\title{
Abordaje de clima organizacional su aplicación en organismos públicos en el marco de la Responsabilidad Social Pública (RSP)
}

Nora Gorrochategui*

Rosana Ferreyra**

Argentina

\section{Resumen}

El trabajo presenta una herramienta de clima organizacional en el marco de la RsP para ser aplicada en diferentes niveles de las organizaciones públicas, el de la alta dirección y la dotación en general. Propone explorar aspectos organizacionales que tiendan a reducir la brecha que se abre entre los principios y materias que abarca la Responsabilidad Social (RS) y su aplicación en la gestión de los recursos humanos de las organizaciones del sector público

\section{Aspectos centrales de estudios de clima laboral en organizaciones públicas}

Se parte de la convicción de que el trabajo es una actividad estructurante, en la cual las personas invierten gran parte de su tiempo, y de

* Doctora en Administración. Universidad de Buenos Aires. Cofundadora del Sirso. Contacto: noragorrochategui@gmail.com

** Magister Scientiarum en Administración Pública. Universidad de Buenos Aires. Contacto: rosana0013@hotmail.com 
que la eficiencia organizacional depende altamente de la calidad de sus recursos humanos. La preocupación por las personas queda inscrita en las más modernas tendencias de gestión vinculadas a la responsabilidad social y a la gestión pública. Baste mencionar algunos ejemplos; el Pacto Global (1999) dedica cuatro principios (3, 4, 5 y 6) a los temas laborales; el Libro Verde de la Unión Europea (2001) destina un capítulo a la gestión de los recursos humanos; la Carta Iberoamericana de la Función Pública (2003) señala al desarrollo del capital humano como condición del buen funcionamiento del servicio público y la iso 26000 (2010) considera a las “prácticas laborales" una materia fundamental y, al mismo tiempo, destaca que la gobernanza de la organización es el factor más importante para hacer que la organización pueda poner en práctica los principios de la responsabilidad social. Así, cuanto más focalizada esté la gestión en la relación existente entre centralidad de la persona asociada a eficiencia organizacional mayor importancia cobran las prácticas de gestión tendientes a proporcionar calidad de vida laboral.

El clima organizacional es una práctica significativa para el estudio de la calidad de vida laboral debido a que su abordaje posibilita identificar indicadores que impactan en forma negativa sobre las personas, el funcionamiento y la gestión de una organización. El análisis de estos indicadores ofrece la posibilidad de revisarlos, diseñar cambios planificados y generar las estrategias de acción pertinentes que colaboren con la mejora de la gestión.

Los beneficios de los estudios de clima plantean al menos dos desafíos previos a su instrumentación, que requieren la reflexión sobre la especificidad de la organización sobre la cual se va a aplicar. Ellos son la identificación de las dimensiones relevantes y la definición del instrumento para recolectar información sobre el clima organizacional.

Respecto al primer desafío, el establecer las dimensiones relevantes, queda evidenciado por la enorme cantidad de elementos que se identifican en investigaciones sobre clima organizacional. Entre los más frecuentemente mencionados en la literatura especializada se destaca el modelo de Lickert, que señala ocho dimensiones: métodos de mando, fuerzas motivacionales y los procesos de comunicación, influencia, toma de decisiones, control y rendimiento y perfeccionamiento. Por su parte, 
Litwin y Stringer identifican seis dimensiones: estructura, responsabilidad individual, remuneración, riesgos y toma de decisiones, apoyo y tolerancia al conflicto. Otros autores coinciden en algunas dimensiones ya enunciadas y se agregan otras, tales como: autonomía, conflicto y cooperación, relaciones sociales, remuneración, estatus, flexibilidad e innovación, apoyo de la alta dirección, apertura a los cambios tecnológicos, tipo de recompensas, consideración y agradecimiento (Sandoval Caraveo, 2004; Orbegoso Galarza, 2010).

En el caso de estudios de clima en organizaciones públicas, las dimensiones a considerar parecen complejizarse aún más, teniendo en cuenta que presentan requerimientos comunes con cualquier tipo de organización y se le agregan dimensiones exclusivas por su carácter público, variando su importancia según el nivel que el funcionario ocupe dentro de la organización. Si bien el establecer categorías de dimensiones mutuamente excluyentes resulta difícil, es posible identificar al menos tres grupos de dimensiones. Un primer grupo, “dimensiones tradicionales", habituales en estudios de clima en cualquier tipo de organización, propias de los niveles operativos de la organización y que afectan el comportamiento del individuo en la organización, tales como el desempeño del puesto de trabajo y las relaciones sociales que se establecen con pares y superiores, a las que se le suman otras, que se destacan por su relevancia para las organizaciones públicas, como por ejemplo, las condiciones del desarrollo de carrera administrativa y la percepción sobre el prestigio social que tiene el desempeño de la actividad pública.

Un segundo grupo, "dimensiones estructurales", que determinan o condicionan el "estar" en el contexto interno de la organización e inciden sobre todos los niveles de la organización, tales como la estructura, la cultura, la plataforma tecnológica, las políticas, el estilo de liderazgo imperante y los valores establecidos.

Un tercer grupo, "dimensiones de gobernanza", nuevas, generadas desde el contexto externo, por el cambio de época vinculadas a la construcción de una sociedad sustentable, corresponden fundamentalmente a los altos niveles de la organización pública y se vinculan a la forma de gestión gubernamental asociados a los principios de la 
responsabilidad social, tales como rendición de cuentas, transparencia, comportamiento ético, respeto a los intereses de las partes interesadas.

La multidimensionalidad del clima organizacional obliga a una selección arbitraria de las dimensiones que sean significativas y relevantes, y que al mismo tiempo aborden la singularidad de la organización en la que se aplican.

En relación con el segundo desafío, definir el instrumento adecuado para medir el clima organizacional, la literatura especializada abunda en reflexiones, que son comunes a las técnicas de recolección de datos frecuentemente utilizadas en investigación, entre las que se destacan la observación directa del comportamiento y desarrollo de los empleados, las entrevistas abiertas y encuestas anónimas. La observación directa requiere que el especialista pueda establecer relaciones informales con los empleados, que en alguna medida, es de difícil instrumentación para quienes son los responsables de la gestión de los recursos humanos en las organizaciones públicas. Las entrevistas en profundidad, por su carácter formal pero no estructurado, permiten profundizar en la conversación con el entrevistado sobre distintos aspectos pautados con base en cuestionarios abiertos, en cuyo desarrollo pueden surgir nuevos elementos a explorar. El cuestionario escrito es una técnica que suministra información confiable, en la medida que se elabore con preguntas cerradas, donde se pida a los miembros de la organización que evalúen la situación actual y cuál sería para ellos la situación ideal, lo que permite conocer la brecha existente entre la realidad que perciben y la deseada. Un aspecto de especial cuidado es el lenguaje que se utilice en los cuestionarios, pues este debe ser acorde a la población encuestada. Entre las técnicas que se utilizan para recolectar información, se suele mencionar con menor frecuencia la organización de sesiones de focus group, que consisten en reuniones con grupos de empleados, dirigidas por un experto que plantea temas asociados al clima incentivando a que los participantes opinen sobre ellos; puede servir de apoyo y complemento a las otras técnicas mencionadas.

Existe una variedad disponible de instrumentos de recolección de información sobre clima organizacional, su aplicación en forma única o combinada dependerá de las características propias y de las circunstancias específicas de la organización pública en las que se apliquen. 
En el caso de los estudios de clima en organizaciones públicas, tanto la consideración de las dimensiones que se seleccionen para su análisis como de los instrumentos de recolección de la información están fuertemente influidos por factores tales como la orientación general del gobierno, la conceptualización de la relación entre funcionarios políticos y planta permanente de la administración pública y la voluntad política de implementar políticas de recursos humanos que contemplen las percepciones de quienes integran esas organizaciones. $\mathrm{Si}$ a esto se suma el hecho de que las nuevas orientaciones de gobernanza moderna contemplan los principios de la responsabilidad social pública, se requiere diseñar estudios de clima organizacional público compatibles con dichas tendencias.

\section{Propuesta de estudio de clima en organizaciones públicas en el marco de la RSP}

La propuesta que se presenta contiene tres componentes vinculados a: 1) la identificación de dimensiones relevantes, 2) el instrumento de recolección de la información y 3) el proceso de implementación del estudio de clima. Tanto las dimensiones como lo relativo al instrumento de recolección de la información varían teniendo en cuenta el nivel del funcionario al que se aplique.

Cabe señalar que la propuesta abarca solamente la preparación del instrumento de recolección de información y la elaboración de los cuestionarios pilotos. Resta efectuar la prueba piloto y elaborar la versión definitiva.

\section{Dotación general del personal}

Se incluyen diez dimensiones en las que se redefinen algunas tradicionales de estudios de clima organizacional a la luz de nuevas situaciones que se desprenden de las relaciones laborales en el ámbito público. Se ofrecen definiciones conceptuales focalizando la atención en lo que se pretende develar. Las dimensiones son: 
1. Sentido de pertenencia/imagen empleado público: explora el grado de satisfacción y orgullo de trabajar en la organización y la valoración social del empleado público.

2. Comunicación interna: explora el grado de eficacia de la comunicación interna.

3. Condiciones de trabajo: explora la calidad de las condiciones de trabajo y el grado en que los empleados cuentan con las herramientas necesarias para la realización de su trabajo.

4. Liderazgo: explora el grado en que los jefes acompañan, estimulan y dan participación a sus colaboradores.

5. Relaciones interpersonales: explora la colaboración, la consideración y el respeto entre pares.

6. Motivación: explora la satisfacción y la capacitación de los empleados en relación con la tarea que realizan.

7. Desarrollo: explora si la organización posee mecanismos formalizados para el crecimiento y desarrollo a los empleados.

8. Conciliación vida familiar y laboral: explora si la organización desarrolla políticas tendientes a conciliar vida familiar y trabajo.

9. Gestión de la diversidad: explora si la organización recluta personas con capacidades diferentes, y/o de diferentes culturas, sin discriminación de género.

10. Retribución: explora el grado de conformidad con remuneración.

El instrumento de recolección de información es una encuesta por muestreo de carácter anónimo. La encuesta consta de preguntas cerradas, con posibilidad de justificación (anexo I). Esto se plantea de esta manera a fin de facilitar la comparabilidad de las respuestas, abriendo la posibilidad de que los agentes opten por la posibilidad de ampliar la información. Teniendo en cuenta que el ambiente de las organizaciones públicas es formalizado, al momento de la administración de la encuesta es necesario garantizar un ambiente tranquilo y que los empleados dispongan del tiempo necesario para realizarla, evitando que los superiores sugieran respuestas o indicaciones que no concuerden con el encuadre. La selección de la fecha es importante siendo recomendable considerar elementos intervinientes en la emocionalidad de los encuestados que predisponga positiva o negativamente en las respuestas, como 
por ejemplo despidos, aumentos salariales y evaluaciones de desempeño. La modalidad de cuestionario autoadministrado, como el que se propone, resulta económico, rápido y de fácil distribución.

\section{Alta dirección}

Las dimensiones del clima organizacional consideradas para ser analizadas por la conducción de los organismos públicos considera algunos de los aspectos relacionados con la gobernanza moderna. El tema de la corrupción, altamente sensible, no se ha incluido para no generar condiciones que impidan la realización del estudio de clima organizacional orientado a la RSP, como es habitual en investigaciones que indagan sobre esta problemática. Las dimensiones seleccionadas son cuatro ${ }^{3}$ :

1. Comportamiento ético: se entiende la existencia de elementos culturales y organizacionales que favorezcan la honestidad, equidad, integridad.

2. Transparencia: se entiende la apertura de la organización para revelar la información sobre políticas y decisiones que afectan a sus partes interesadas.

3. Relación con las partes interesadas: se entiende la existencia de procedimientos de interacción formal de la organización con sus partes interesadas en todas las etapas de la gestión gubernamental (planificación, implementación y control) que permitan considerar sus requerimientos en función de su relevancia, exhaustividad e impactos.

4. Rendición de cuentas: se entiende las justificaciones y explicaciones que el funcionario público brinda al hacerse responsable, ante sí mismo, una autoridad y partes interesadas, por

3 Un análisis conceptual detallado de las dimensiones de RSP sobre clima organizacional se pueden ver en Gorrochategui (2016). Las dimensiones del análisis del clima organizacional en el marco de la Responsabilidad Social Pública (RSP). Ciencias Administrativas Año 4, N. ${ }^{\circ}$ 7. Enero/junio 2016. Recuperado de http://revistas.unlp.edu.ar/CADM 
las acciones u omisiones en el desempeño de las responsabilidades que le han sido conferidas al ser designado en un cargo.

El instrumento de recolección de información es una entrevista, con base en un cuestionario estructurado con preguntas cerradas (anexo II), en las que eventualmente se podrán incorporar algunas preguntas abiertas, según la organización, lo que permitiría obtener un conocimiento más acabado sobre algún aspecto específico, en el que el entrevistado desee explayarse.

\section{Proceso de implementación del estudio de clima}

Se sugieren las siguientes fases:

Fase 1. Prediagnóstico: consiste en relevar, a partir del consentimiento expreso de los responsables máximos de la organización pública, información general sobre la organización, atravesamientos culturales, estilos de dirección, políticas, quejas y demandas del personal. Se investiga con profundidad antecedentes sobre las encuestas de clima anteriores, la forma como fueron llevadas a cabo, qué porcentaje de la población participó, si hubo devolución posterior a la intervención en campo y si se concretó algún plan de acción.

Fase 2. Talleres de sensibilización: consiste en la promoción e impulso del proceso de comunicación a los empleados, previo a la administración de las encuestas, sobre el contenido y alcance de la encuesta, los beneficios, la explicación del proceso previo, durante y posterior a la encuesta y la explicitación de los compromisos asumidos por la organización con los empleados. Es pertinente que directivos de la organización se involucren en el proceso de sensibilización y sean quienes soliciten el apoyo y el compromiso de los colaboradores y el personal en su conjunto durante todo el proceso.

Fase 3. Diseño y adecuación de encuesta de clima laboral: consiste en la adecuación de los cuestionarios (anexos I y II), con 
base en la información relevada en la fase 1, que consideren la especificidad de la organización pública en la que se aplique. Se sugiere no relevar y generar expectativas sobre problemáticas que la dirección no esté en condiciones de modificar, dado que frecuentemente las máximas autoridades de las organizaciones públicas no están en condiciones de introducir modificaciones sobre cuestiones estructurales relativas a las políticas de recursos humanos, pero sí sobre lo vinculado a la gestión organizativa.

Fase 4. Aplicación de la encuesta/entrevista: consiste en velar por la naturaleza anónima de la encuesta, las características del ambiente en el que esta se administra y en el control de condiciones de diversa índole que puedan incidir en las respuestas que se ofrezcan.

Fase 5. Análisis e interpretación de resultados: consiste en la realización de operaciones que hallan propiedades y relaciones que surgen de los datos e interpretarlos considerando no solo los resultados globales a nivel de organización, sino considerar los que se produzcan por direcciones y áreas, con el propósito de que los planes de acción que se propongan alcancen a todos los niveles de la estructura organizacional.

Fase 6. Diagnóstico y propuesta de estrategias de acción: Las estrategias de acción inferidas del diagnóstico de situación deben trabajarse con los líderes de cada dirección/área, para establecer propuestas de mejora conjuntas, consensuadas y factibles de aplicación. Los planes deben informarse a la brevedad de la publicación de los resultados para evidenciar el compromiso asumido por la alta dirección en el proceso.

\section{Conclusiones}

La RSP está comenzando a formalizarse en la administración pública, fundamentalmente, a partir de la introducción de los principios y materias en la legislación nacional de los países de América Latina. Sin embargo, al interior de las organizaciones públicas a pesar de la importancia que las recomendaciones internacionales asignan al capital 
Responsabilidad Social de las Organizaciones (RSO)

humano, las tecnologías para incorporar la RSP todavía es incipiente. En tal sentido, la propuesta sobre estudio del clima organizacional en el marco de la RSP se considera una innovación tendiente a la concreción de la gobernanza moderna. 


\section{Referencias}

Carta Iberoamericana de la Función Pública (2003). Recuperado de http:// www.sgp.gov.ar/contenidos/cofefup/documentos/docs/Carta_Iberoamerica_de_la_Funcion_Publica.pdf

Chiang, M., Salazar Botello, C., Huerta Rivera, P. y Núñez Partido, A. (2008). Clima organizacional y satisfacción laboral en organizaciones del sector estatal (Instituciones públicas). Desarrollo, adaptación y validación de instrumentos. Revista Universum, 23(2), 66-85. Recuperado de http:// www.scielo.cl/scielo

Comisión de las Comunidades Europeas (2001). Libro verde. Fomentar un marco europeo para la responsabilidad social de las empresas. Bruselas. Recuperado de http://eur-lex.europa.eu/legal-content/ES/TXT/ PDF/?uri=CELEX:52001DC0366\&from=ES

Gorrochategui, N. (Enero-junio, 2016). Las dimensiones del análisis del clima organizacional en el marco de la responsabilidad social pública (RSP). Ciencias Administrativas, Año 4, N. ${ }^{\circ}$ 7. Recuperado de http://revistas. unlp.edu.ar/CADM

IRAM-ISO 26000 (2010). Guía de Responsabilidad Social. Buenos Aires.

Orbegoso Galarza, A. (2010). Problemas teóricos del clima organizacional: un estado de la cuestión. Revista Psicología, 12, 347-362. Recuperado de http://ojs.ucv.edu.pe/index.php/R_PSI/article/view/341

Pacto Global (1999). Principios del Pacto Global. Recuperado de https:// www.unglobalcompact.org/

Sandoval Caraveo, M. (2004). Concepto y dimensiones del clima organizacional. Hitos de Ciencias Económico Administrativas. Hitos de Ciencias Económico Administrativas, 10(27), 78-82. Mayo-agosto 2004. Recuperado de www.academia.edu/9225037/ 


\section{Anexo I}

\section{Encuesta para la dotación}

El organismo tiene como objetivo explorar el clima laboral y relevar las necesidades de los empleados mediante esta encuesta, a fin de mejorar la calidad de vida en el trabajo y el bienestar del personal.

La encuesta es anónima y la información relevada confidencial.

Dirección a la que pertenece:

Área/Sector de trabajo:

Antigüedad en el puesto actual (Señalar la opción que corresponda) Hasta 5 años $\bigcirc$ Entre 6 y 10 años $\bigcirc$ Más de 11 años $\bigcirc$

\section{a. Organismo}

¿Es de su agrado la imagen que el organismo proyecta en la comunidad?

Justifique

¿Se siente orgulloso de pertenecer a este organismo?

Justifique

¿Conoce la Visión, Misión, Valores del organismo?

Justifique

3- ¿Considera que la imagen de empleado público de este organismo es positiva en la comunidad?

Justifique 


\section{b. Comunicación interna}

$¿$ Los directores comunican los objetivos y estrategias del organismo?

Justifique

6- ¿La dirección comunica las razones de los cambios que realizan? Justifique

7- ¿Se enteró de lo que ocurre en la organización por la comunicación oficial?

Justifique

\section{c. Condiciones de trabajo}

8- ¿El espacio físico donde desempeña sus tareas es agradable?

Justifique

9- ¿Las condiciones ambientales (limpieza, temperatura, iluminación, comodidad, ruido, ventilación, etc.) son adecuadas?

Justifique

10- ¿Las herramientas de trabajo necesarias para realizar la tarea (computadoras, escritorios, artículos de librería, etc.) son apropiadas?

Justifique

\section{d. Liderazgo}

Sobre superiores inmediatos (coordinador/supervisor)

11- ¿Su superior crea un ambiente de confianza y colaboración en el grupo de trabajo?

Justifique

12 - ¿Comunican de manera clara y oportuna, cuáles son las funciones de su puesto y sus responsabilidades?

Justifique 
13- ¿Escucha aportes y sugerencias de sus colaboradores y los considera?

Justifique

14- ¿La distribución de la tarea es equitativa en su área?

\section{e. Relaciones Interpersonales}

15- ¿Las relaciones con sus compañeros de trabajo son cordiales? Justifique

16- ¿Considera que en su área se trabaja en equipo exitosamente? Justifique

17- ¿Existe colaboración y un clima de trabajo agradable entre compañeros en su área de trabajo?

Justifique

\section{f. Motivación}

18- ¿Es de su agrado la tarea que realiza?

Justifique

19- ¿Recibe algún tipo de reconocimiento de parte de sus superiores directos cuando alcanza los objetivos de su puesto?

Justifique

20- ¿Considera que posee la capacitación necesaria para desempeñarse en su trabajo?

Justifique

\section{g. Desarrollo}

21- ¿Considera que el organismo ofrece oportunidades de crecimiento y desarrollo formalizadas?

Justifique 
22- ¿Las oportunidades de crecimiento y progreso se asigna a los empleados con conocimiento y mérito?

Justifique

23- ¿Se administran evaluaciones de desempeño en la organización? Justifique

24- ¿Considera que se tienen en cuanta las evaluaciones de desempeño para ofrecer oportunidades de crecimiento y desarrollo? Justifique

\section{h. Conciliación vida familiar y laboral}

25- ¿Ante necesidades operativas el organismo extiende su jornada laboral?

Justifique

26- ¿Si un empleado posee algún compromiso familia (reunión de padres, adaptaciones escolares, cumpleaños, etc.) el organismo cuenta con políticas flexibles para atender estas necesidades?

Justifique

27- ¿Considera que existe en el organismo una cultura organizacional, orientada a generar prácticas de conciliación entre la vida laboral y la familiar?

Justifique

\section{i. Gestión de la diversidad}

28- ¿Considera que en el organismo hay políticas de selección que incluya a personas con capacidades diferentes?

Justifique

29- ¿Considera que posee la capacitación necesaria para desempeñarse en su trabajo?

Justifique 
Responsabilidad Social de las Organizaciones (RSO)

\section{j. Retribución}

30- ¿Considera que su remuneración comparada con otros organismos es acorde a las responsabilidades de su puesto?

Justifique

31- ¿Está conforme con la remuneración recibida?

Justifique

Muchas gracias por su tiempo y colaboración 


\section{Anexo II}

\section{Cuestionario para la entrevista con la Alta Dirección}

Comportamiento ético: se entiende la existencia de elementos culturales y organizacionales que favorezcan la honestidad, equidad e integridad.

1. ¿Puede identificar en la organización tradiciones, costumbres, usos que sean compartidos y que reflejen una cultura orientada por valores que favorezcan la honestidad, equidad e integridad?

2. ¿Los valores de la organización son públicos, conocidos por todos los empleados e incorporados en los comportamientos de los integrantes de la organización?

3. ¿Cuenta la organización con un código ético?

4. ¿Se capacita a los empleados sobre la ética de la función pública?

Transparencia: se entiende la apertura de la organización para revelar la información sobre políticas y decisiones que afectan a sus partes interesadas.

5. ¿Considera que los empleados están concientizados sobre la misión, visión y políticas del organismo?

6. ¿Cuenta la organización con procesos de difusión de los objetivos estratégicos?

7. ¿La organización produce algún documento en el que informa regularmente sobre los resultados de su gestión en las dimensiones económico-financiera, social y medioambiental?

Relación con las partes interesadas: se entiende la existencia de procedimientos de interacción formal de la organización con sus partes interesadas en todas las etapas de la gestión gubernamental (planificación, implementación y control) que permitan considerar sus requerimientos en función de su relevancia, exhaustividad e impactos (beneficiarios, clientes, proveedores, otros organismos públicos, empresas, organizaciones no gubernamentales, sindicatos, cámaras). 
8. ¿Cuenta la organización con mecanismos organizacionales para involucrar a las partes interesadas en el proceso de gestión de la organización?

(Conversaciones con las partes interesadas, diálogos abiertos, encuestas, consultas telefónicas, quejas)

9. ¿El organismo posee una metodología que mida el impacto de las políticas, programas, acciones y servicios ofrecidos a los beneficiarios que contribuyan a la mejora de los mismos?

Rendición de cuentas: se entiende las justificaciones y explicaciones que el funcionario público brinda al hacerse responsable, ante una autoridad y ante sí mismo, por las acciones u omisiones en el desempeño de las responsabilidades que le han sido conferidas al ser designado en un cargo.

10. ¿Entre sus funciones, se le solicita dar explicaciones ante alguna autoridad, de manera formal, acerca del desempeño de su trabajo, a través de mecanismos claramente definidos?

11. ¿Cuenta con sistemas de información confiables y accesibles que le permitan mostrar los resultados de su gestión? ¿Cuáles? 